\title{
Shared Space Modeling Based on Social Forces and Distance Potential Field
}

\author{
Bani Anvari ${ }^{1}$, Winnie Daamen ${ }^{2}$ Victor L. Knoop ${ }^{2}$, Serge P. Hoogendoorn ${ }^{2}$, and \\ Michael G.H. Bell ${ }^{1}$ \\ ${ }^{1}$ Centre for Transport Studies, Department of Civil and Environmental Engineering, Imperial \\ College London, SW7 2BU, United Kingdom, \\ b.anvari09eimperial.ac.uk \\ 2 Transport \& Planning, Delft University of Technology, The Netherlands
}

\begin{abstract}
Urban design is moving towards space sharing in order to increase the community texture and safety of street surroundings. However, there is a need for a simulation tool capable of representing future shared space schemes to help judging the designs under which shared space design is a suitable alternative to traditional street designs. This paper presents a microscopic mathematical model that is used a traffic simulation tool capable to represem main behaviors of pedestrians and cars in any shared space layout. This is achieved by generating a route map which helps agents to find the shortest path towards their target destinations on the strategic level. On the operational level, the Social Force Model (SFM) is used and extended for a mixed traffic to produce feasible trajectories. The trajectory results are presented to give a face-validation of the functionality of the shared space simulation model.
\end{abstract}

Key words: shared space, Social Force Model, flood fill algorithm

\section{Introduction}

Shared space is about changing the way streets function through reducing the dominance of vehicles. This is often obtained through the use of a shared surface street, which itself aims at integrated space use [3]. Shared space allocates more degrees of freedom to its traffic participants. As a result of this increased freedom, traffic engineers became concerned about ensuring safe and efficient movement of pedestrians and drivers in these shared schemes. Computer modeling does permit an approach towards an operational field evaluation for future shared space designs while describing the behavior of traffic participants. A combination of two traffic systems requires way of finding the path to a destination between different traffic modes, handling social interactions through the shared area and also the propulsion typical for each mode. Many theoretical models have been proposed so as to uncover the laws which govern both vehicle traffic as well as pedestrian dynamics [17], [5], [2]. In regards to the analysis of pedestrian behavior using methods from physics, the Social Force Model (SFM) is one of the most discussed models on the microscopic level [15], [13], [16]. The SFM has the potential to describe gap acceptance behaviour emergently. It offers the possibility of a unified theory that can explain both vehicle and pedestrian movement, both separately 
and in interaction with each other.

A literature review on mixed traffic is given in Section 2. The main behavior of shared space users is explained based on social forces in Section 3. The shortest path to the destination is generated based on the flood fill algorithm for each shared space user here (Section 4). Finally, Section 5 illustrates the ability of the mathematical model through qualitative examination of the performed simulations.

\section{Background}

Concerning mixed pedestrian-vehicle flows over a network, limited numbers of studies have been reported in the literature. Among pedestrian and vehicle interaction models, Helbing [8] formulated and analyzed the interaction of pedestrians with vehicles on the crossing sections and Jiang [11] explored a lattice gas model in order to study the vehicle and pedestrian flows in a narrow channel. The pedestrian traffic is studied in [10], [1] using VISSIM and vehicle and pedestrian modes are operated independently and controlled by the traffic signals at potential conflicting areas. Apart from shared space scenarios, the interaction of pedestrians and vehicles was modeled on the microscopic level by using the SFM for pedestrian behavior, the single track model for car dynamics and a game theoretic force for solving conflicts in [14].

This paper addresses modeling mixed traffic environments with the use of the SFM. Social forces are used for both pedestrian and car dynamics. The SFM is formulated in such a way that shared space users avoid collisions and interruptions within their personal space. Simulations of the SFM show that it is essential to integrate a shortest path algorithm in order to model a human-like behavior. Potential field methods [12] and related path modification methods [4] have been a popular choice, due to their relative ease and effectiveness at coordinating motion. A distance map based on a combination of Manhattan Metric and Chessboard Metric is used in this paper to navigate shared space users into the direction of the shortest path to their goal according to the infrastructure of the environment.

\section{A Microscopic Model for Shared Space Users}

The microscopic model presented in this section is based on Helbing's SFM [7]. The new arrangement of a shared space environment integrating vehicles is shown in Figure 1 . The sum of the force terms exerted to a car $\gamma$ from a pedestrian $\alpha$, a boundary $b$ and another vehicle $\delta$ can be seen in Equation 1. The last term is a random fluctuation force exerted to the sum of the forces to show the velocity fluctuation due to diverse behaviors. Each summand will be explained in the following sections.

$$
\frac{d \mathbf{v}_{\gamma}(t)}{d t}=\mathbf{f}_{\gamma}^{0}+\sum_{\delta(\delta \neq \gamma)} \mathbf{f}_{\gamma \delta}+\sum_{\alpha} \mathbf{f}_{\gamma \alpha}+\sum_{b} \mathbf{f}_{\gamma b}+\xi
$$




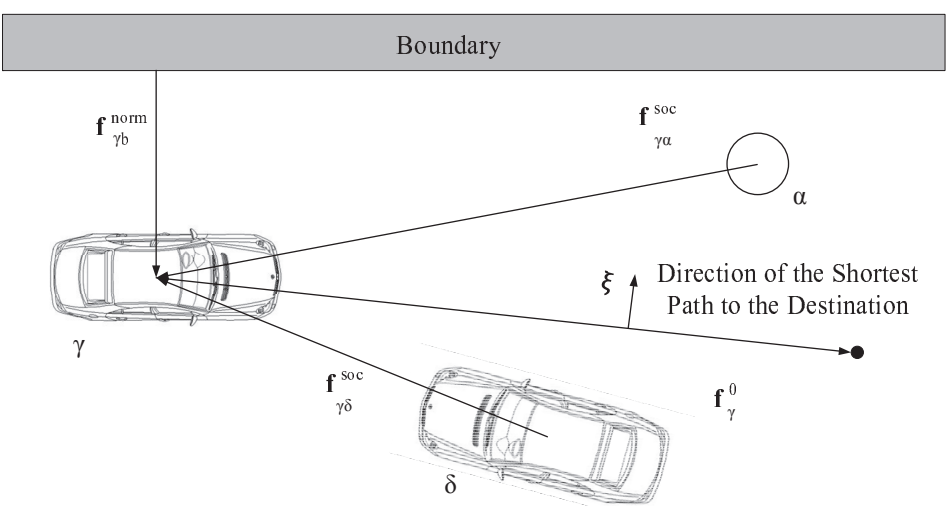

Fig. 1: Force Terms Exerted to a Car from a Pedestrian/Car/Boundary

\subsection{Driving Force}

The driving force of a car is similar to the one applied for pedestrians as this force term describes the motivation to move towards a certain destination. The driver $\gamma$ is assumed to move in a desired direction $\mathbf{e}_{\gamma}^{0}$ with a desired speed $v_{\gamma}^{0}$ that is adapted to the actual velocity $\mathbf{v}_{\gamma}$ within a certain relaxation time $\tau_{\gamma}$.

$$
\mathbf{f}_{\gamma}^{0}=\frac{v_{\gamma}^{0} \cdot \mathbf{e}_{\gamma}^{0}(t)-\mathbf{v}_{\gamma}}{\tau_{\gamma}}
$$

The desired direction $\mathbf{e}_{\gamma}^{0}$ above points into the direction of the shortest path to the destination. This is achieved by calculating a distance map that is explained in Section 4.

\subsection{Interaction Forces Considering the Geometric Model of Cars}

Shared space layouts are aiming to make a smooth traffic flow through reducing the stop and go behavior. Drivers try adapting to the behavior of other shared space users. Any deviation from their path to their destination is mainly due to collision avoiding interactions. The interaction between a car $\gamma$, either with another $\operatorname{car}(U=\delta)$ or with a pedestrian $(X=\alpha)$ is described in Equation 3. The socio-psychological force $\mathbf{f}_{\gamma U}^{\text {soc }}$ is to keep a certain distance from nearby users. The deceleration force $\mathbf{f}_{\gamma \alpha}^{\mathrm{dec}}$ is to cover the follow-the-leader behavior of drivers if a car is faster than a leading car within a close distance. Assuming no physical contact, vehicles do not get closer than a certain distance to another agent. Hence, no physical force is included.

$$
\mathbf{f}_{\gamma U}(t)=\mathbf{f}_{\gamma U}^{\mathrm{soc}}(t)+\mathbf{f}_{\gamma \delta}^{\mathrm{dec}}(t)
$$

To describe the socio-psychological force $\mathbf{f}_{\gamma U}^{\text {soc }}$, an exponential function is applied to reflect the role of distance. The repulsive force increases when agents get closer and it will almost vanish when they move far away from each other.

$$
\mathbf{f}_{\gamma U}^{\mathrm{soc}}=A_{\gamma U} e^{\frac{r_{\gamma U}-d_{\gamma U}}{B_{\gamma U}}} \mathbf{n}_{\gamma U} F_{\gamma U}
$$


The $\mathbf{n}_{\gamma U}$ is the normalized vector pointing from another user (car or pedestrian) to the car $\gamma . A_{\gamma U}$ and $B_{\gamma U}$ are constant parameters that indicate interaction strength and interaction range of the repulsive force $\mathbf{f}_{\gamma U}^{\mathrm{soc}} \cdot d_{\gamma U}$ is the distance between the center of agents, and $r_{\gamma U}$ is the sum of their radii. Since the form factor and the radii are different from the one for pedestrians, these factors are redefined.

Determination of the Sum of Radii: Contrary to a circular specification for a pedestrian in the SFM, an ellipse with radius $r_{\gamma}$ is modeled for a car (Figure 2). The radius $r_{\gamma}$ depends on the angle $\varphi_{\gamma U}$ between the desired direction of a car and the direction of the close-by pedestrian or car who is exerting a force. The radius of the ellipse $r_{\gamma}\left(\varphi_{\gamma U}\right)$ in polar coordinates is as follows:

$$
r_{\gamma}\left(\varphi_{\gamma U}\right)=\frac{w}{\sqrt{1-\varepsilon^{2} \cos ^{2}\left(\varphi_{\gamma U}\right)}}, \text { where } \varepsilon=\frac{\sqrt{l^{2}-w^{2}}}{l}
$$

In Equation 5, $2 l$ and $2 w$ are assumed to be the average length and width of a modeled

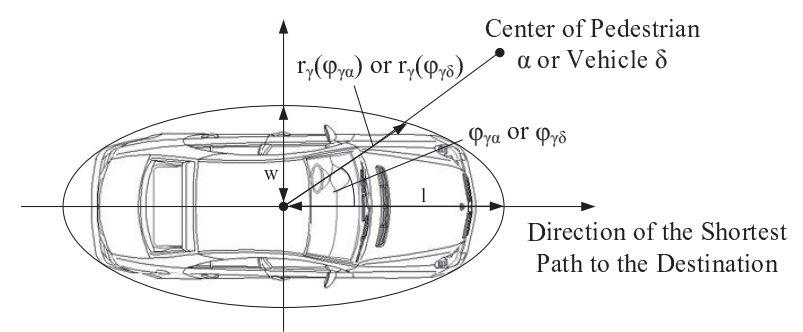

Fig. 2: Vehicle Modeling using a Geometrical Approximation of an Ellipse

car. The sum of the radii is $r_{\gamma U}=r_{\gamma}+r_{U}$ and $d_{\gamma U}$ is the distance between the center of a car $\gamma$ and another shared space user (car or pedestrian). The form factor $F_{\gamma U}$ (Equation 6) is used only to consider the anisotropic behavior of human beings. Considering that a car movement is restricted to change of direction, and lateral movement is not possible, an effective field of view is included in this form factor. In addition, there is a difference between a pedestrian and a car following another car since the leading car driver will not only react on the cars in front but also to the cars behind it.

$$
F_{\gamma U}=\left(\lambda_{\gamma}+\left(1-\lambda_{\gamma}\right) \frac{1+\cos \left(\varphi_{\gamma U}\right)}{2}\right) \cdot q
$$

$q$ is the 'effective factor' that will distinguish between a car-pedestrian or a car-car interaction. The driver's view angle is compared to the effective field of view in Figure 3.

Car-following Feature: The deceleration force $\mathbf{f}_{\gamma \delta}^{\mathrm{dec}}$ in Equation 3 is considered for drivers in the same direction of movement within a certain distance to adopt the behavior of the leading car. The social force $\mathbf{f}_{\gamma \delta}^{\text {soc }}$ is assigned zero in this case.

The deceleration force $\mathbf{f}_{\gamma-1, \gamma}^{\text {dec }}$ is defined in Equation 7 [9] and its magnitude depends on the distance between cars $d_{\gamma \delta}$ considering the velocity dependence safe distance $d\left(v_{\gamma} \delta\right)$, velocity differences $\Delta \mathbf{v}_{\gamma \delta}$ and braking time $\tau_{\gamma}^{\prime}$. 


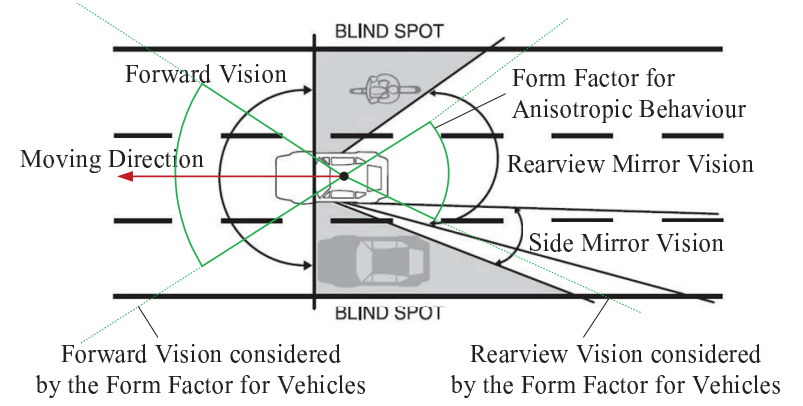

Fig. 3: Effective Field of View Compared to Driver's Vision

$$
\mathbf{f}_{\gamma \delta}^{\mathrm{dec}}=\frac{\Delta \mathbf{v}_{\gamma \delta} \Theta\left(\Delta v_{\gamma \delta}\right)}{\tau_{\gamma}^{\prime}} e^{-\left[\frac{d_{\gamma \delta}-d\left(v_{\gamma}\right)}{B_{\gamma \delta}}\right]} \cdot p, \text { where }\left\{\begin{array}{l}
\Theta\left(\Delta v_{\gamma \delta}\right)=1, \text { if }\left(v_{\gamma}>v_{\delta}\right) \\
\Theta\left(\Delta v_{\gamma \delta}\right)=0, \text { otherwise }
\end{array}\right.
$$

The angle between the desired direction of a driver $\gamma$ and the desired direction of the driver in front of him $\delta$ determines whether their movements are confluent or opposed. Therefore, the deceleration force is only included $(p=1)$ in the sum of the forces when $\left|\varphi_{\gamma \delta}\right| \succ 10^{\circ}$ or $\left|\varphi_{\gamma \delta}+\varphi_{\delta \gamma}-180\right| \prec 10^{\circ}$. Otherwise, $p=0$ and the social force will result in collision avoidance behavior.

The interaction between cars and boundaries/obstacles is described by considering that cars are not expected to have any physical contact with boundaries or obstacles. An expression similar to Equation 4 is defined to avoid car accidents.

\subsection{Relation between Steering Angle and Moving Velocity}

As mentioned in Section 3.2, vehicles have restricted lateral movement. These limitations are included within the model. Regarding pedestrians, the angle of movement can be within $[0,2 \pi]$, whereas the desired velocity should be $v_{\alpha}(t)=5.4 \frac{\mathrm{km}}{\mathrm{h}}$. In respect of cars, not only the velocity is restricted to $20 \frac{\text { miles }}{\mathrm{h}}\left(32 \frac{\mathrm{km}}{\mathrm{h}}\right)$, but also the angle of steering is limited. As shown in Figure 4a, the limitation function can be described as $v_{\text {yempirical }}\left(\psi_{\text {car }}\right)$, where $\psi=\arctan \left(\frac{v_{\gamma 2}}{v_{\gamma 1}}\right)$ is called the steering angle. This function is assumed based on obsereved behaviors. Figure $4 \mathrm{~b}$ illustrates the same concept in polar coordinates and Equation 1 gives the resulting angle of movement and the velocity by computing $\mathbf{v}_{\gamma}(t)$. So, it is necessary to constrain Equation 1 . The steering angle $\psi$ is considered to be within the interval $\left[0, \pm 30^{\circ}\right]$, while $v_{\gamma}(t)$ is considered not to exceed $32 \frac{\mathrm{km}}{\mathrm{h}}$.

\subsection{SFM Adjustment and Extension for Pedestrians}

Since the original SFM [6] only considers forces exerted by pedestrians and obstacles onto other pedestrians, forces exerted by vehicles onto pedestrians need to be included as well. The existence of cars in a shared space environment is expressed by a new socio-repulsive force $\mathbf{f}_{\alpha \gamma}$ term from cars to a pedestrian (Equation 8). This new force 


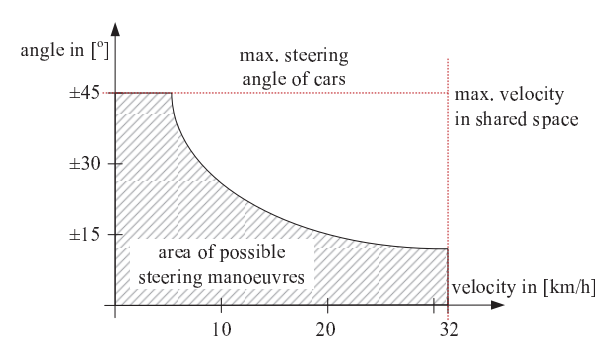

(a) Velocity versus Steering Angle

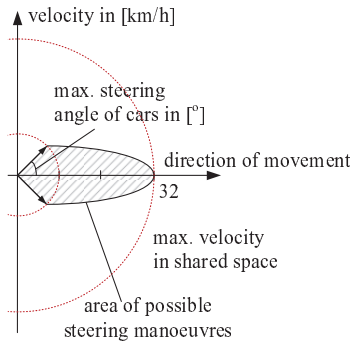

(b) Steering Manoeuvres in Polar

Coordinates

Fig. 4: Vehicle Velocity versus Steering Angle

explains the most important interaction behavior of a pedestrian keeping a certain distance to the nearby car since no physical interaction should occur.

$$
\frac{d \mathbf{v}_{\alpha}(t)}{d t}=\mathbf{f}_{\alpha}^{0}+\sum_{\beta(\beta \neq \alpha)} \mathbf{f}_{\alpha \beta}+\sum_{b} \mathbf{f}_{\alpha b}+\sum_{\gamma} \mathbf{f}_{\alpha \gamma}+\xi
$$

Similar to the interaction force between pedestrians in the Social Force Model, an exponential function is applied to pedestrian $\alpha$ to represent the influence of distance between the pedestrian and the close-by car $\gamma$ as

$$
\mathbf{f}_{\alpha \gamma}^{s o c}=A_{\alpha \gamma} e^{\frac{r \alpha \gamma-d_{\alpha \gamma}}{B \alpha \gamma}} \mathbf{n}_{\alpha \gamma} F_{\alpha \gamma},
$$

where $r_{\alpha \gamma}=r_{\alpha}+r_{\gamma} ; d_{\alpha \gamma}$ is the distance between the center of pedestrian $\alpha$ and car $\gamma$; $\mathbf{n}_{\alpha \gamma}$ is the normalized vector from car $\gamma$ to pedestrian $\alpha$. The form factor $F_{\alpha \gamma}$ is also set similar to Equation 6 to explain the anisotropic behavior of pedestrian $\alpha$ when facing $\operatorname{car} \gamma$.

\section{Desired Direction of Movement by Distance Map}

The direction of the desired velocity is meant to be equivalent to the direction of the shortest path to the destination avoiding obstacles. A distance potential field is calculated by the flood fill algorithm for agents' navigation. The floor area is divided into cells of size $15 \times 15 \mathrm{~cm}^{2}$. Agents move to one of the eight neighboring cells with the closest distance to the target in each time step. This is achieved by calculating the flood fills based on a combination of Manhattan metric $D^{\mathrm{M}}$ and Chessboard metric $D^{\mathrm{C}}$ [12]:

$$
D^{V 2}=(\sqrt{2}-1) D^{m}+D^{C}, \text { where }\left\{\begin{array}{l}
D^{\mathrm{M}}=\sum_{i}\left|\delta x_{i}\right|+\sum_{i}\left|\delta y_{i}\right| \\
D^{\mathrm{C}}=\sum_{i} \max \left(\left|\delta x_{i}\right|,\left|\delta y_{i}\right|\right) \\
D^{\mathrm{m}}=D^{\mathrm{M}}-D^{\mathrm{C}}
\end{array}\right.
$$

\section{Simulation and Results}

This section simulates the presented model to show the behavior of drivers and pedestrians with the car-following feature and the applied distance map. The scenario in 
Figure 5 show the influence of the deceleration force $\mathbf{f}_{\gamma \delta}^{\mathrm{dec}}$ explained in Section 3.2. The interaction force improves the driver behavior in regards to the leading car. Figure 6 illustrates the influence of the distance map on pedestrian route choice behavior based on the flood fill algorithm. The pedestrian therefore chooses a more human-like route after generating a distance map.

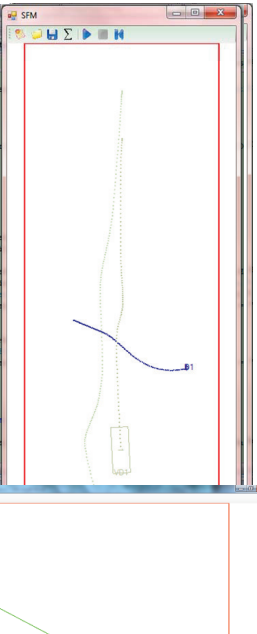

(a)

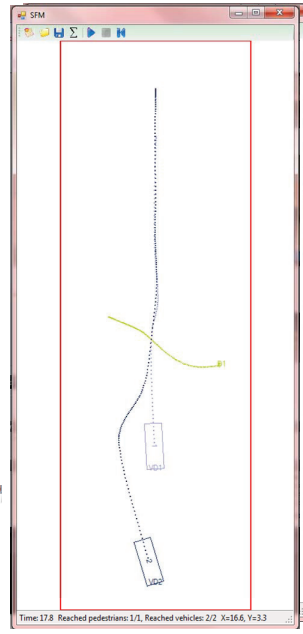

(b)

Fig. 5: Snapshot of a Driver's Path with the Influence of (a) Social Force (SF) and (b) Deceleration Force (DC): The moving direction of the cars is from top to bottom whereas a pedestrian crosses from left to right.

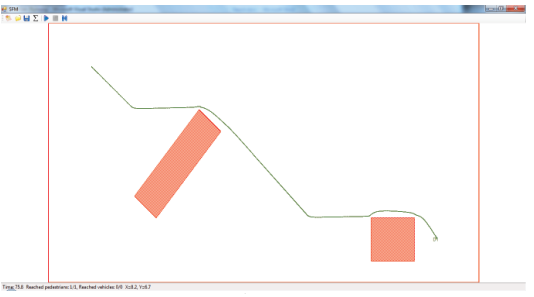

(a) Without Route Map

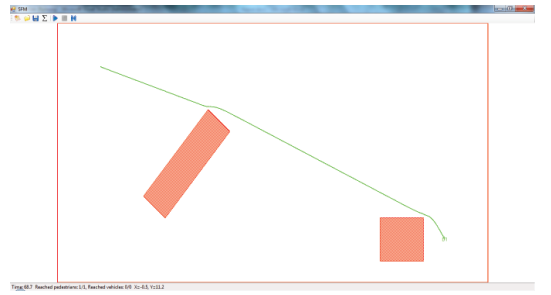

(b) With Route Map

Fig. 6: Obstacle Avoidance and Way-finding Simulation

\section{Conclusions and Future Work}

The main contribution of this paper is the integration of the car-following model and a shortest path algorithm into the SFM. By merging these models, shared space dynamics can be modeled and simulated. The experimental results show that the flood fill algorithm is effective and a feasible option for improving the path planning of the SFM as it provides a more human-like route-choice behavior. The mathematical model is flexible and includes the main interaction factors between drivers and pedestrians in mixed 
traffic environments. For future applications, this model should be calibrated based on real data comparison.

\section{Acknowledgement}

The authors would like to thank NEARCTIS for the financial support of this study under grant number 224272 .

\section{References}

1. C. Boenisch and T. Kretz. Simulation of Pedestrians Crossing a Street. In Traffic and Granular Flow' '09, 2009.

2. M. Chraibi and A. Seyfried. Generalized Centrifugal-Force Model for Pedestrian Dynamics. Physical Review E, 82:046111, 2010.

3. DfT. Shared Space. Technical report, TSO, October 2011.

4. E.W. Dijkstra. A Note on Two Problems in Connexion with Graphs. Numerische Mathematik, 1:269271, 1959.

5. R.S. Franca, M.G.B. Marietto, W.R. Santana, and G. Kobayashi. An Agent-Based Simulation Model for Pedestrian Unidirectional Movement. In Second International Conference on the Applications of Digital Information and Web Technologies, 2009.

6. D. Helbing. A Mathematical Model for the Behaviour of Pedestrians. Behavioral Science, 36:298-310, 1991.

7. D. Helbing, I. Farkas, and T. Vicsek. Simulating Dynamical Features of Escape Panic. $\mathrm{Na}$ ture, 407:487-490, 2000.

8. D. Helbing, R. Jiang, and M. Treiber. Analytical Investigation of Oscillations in Intersecting Flows of Pedestrian and Vehicle Traffic. Physical Review E, 72:0461301-04613010, 2005.

9. D Helbing and B Tilch. Generalized Force Model of Traffic Dynamics. Physical Review E, 58:133-138, 1999 .

10. M.M. Ishaque and R.B. Noland. Trade-offs between Vehicular and Pedestrian Traffic using Micro-Simulation Methods. Transport Polcy 14, 124:124138, 2007.

11. R. Jiang and Q.S. Wu. Interaction between Vehicle and Pedestrians in a Narrow Channel. Physica A, 364:239246, 2006.

12. T. Kretz, C. Bonisch, and P. Vortisch. Comparison of Various Methods for the Calculation of the Distance Potential Field. Pedestrian and Evacuation Dynamics, 2008.

13. M. Moussaid, D. Helbing, S. Garnier, A. Johansson, M. Combe, and G. Theraulaz. Experimental Study of the Behavioural Mechanisms Underlying Self-Organization in Human Crowds. Proceedings of the Royal Society B, 276:2755-2762, 2009.

14. R. Schnauer, M. Stubenschrott, W. Huang, C. Rudloff, and M. Fellendorf. Modeling Concepts for Mixed Traffic: Steps towards a Microscopic Simulation Tool for Shared Space Zones. In TRB 2012 Annual Meeting, 2012.

15. A. Seyfried, B. Steffen, W. Klingsch, and M. Boltes. The Fundamental Diagram of Pedestrian Movement Revisited. Statistical Mechanics: Theory and Experiment, 10:P10002, 2005.

16. Z. Zainuddin and M. Shuaib. Incorporating Decision Making Capability into the Social Force Model in Unidirectional Flow. Research Journal of Applied Science 5, 6:388-393, 2010.

17. H.M. Zhang and T. Kim. A Car-following Theory for Multiphase Vehicular Traffic Flow. Transport Research Part B, 39:385-399, 2005. 